\title{
Dysthymia Characteristic of Heavy Alcohol Use in Depressed Adolescents
}

\author{
Cheryl A. King, Michael W. Naylor, Elizabeth M. Hill, Benjamin N. Shain, and \\ John F. Greden
}

\section{Introduction}

The increased prevalence of heavy alcohol use and abuse among depressed adolescents has been well documented (as reviewed in Bukstein et al 1989). As an example, Greenbaum et al (1991; studied 547 adolescents having serious emotional disturbances, and found that adolescents with severe depressive disorders were more likely to be diagnosed with a substance-abuse disorder, including alcohol abuse/dependency, than were adolescents without depressive disorders (48.3\% versus $17.0 \%)$. A high prevalence of alcohol abuse in depressed adolescents also has been documented in nonclinical samples (Deykin et al 1987).

Recognition and appropriate treatment of alcohol abuse in depressed adolescents are critical. Both alcohol abuse and dejression tend to be chronic conditions with high rates of secondary problems. The co-occurrence of alcohol abuse and depression is particularly problematic, having been associated with an increased risk for suicidal behavior in adolescents (King et al 1993; Pfeffer et al 1988). Despite the importance of identifying and treating each of these conditions, common clinical features make differentiation difficult. Studies with adults suggest that depression may actually motivate treatment-seeking in alcohoi abusers (Regier et al 1990), but that the alcohol abuse often goes unrecognized by treatment providers (Helzer and Pryzbeck 1988). The recognition of alcohol-abuse patterns in depressed adolescents would be assisted by information on the phenomenology of depression in adolescents who abuse alcohol. This study was conducted to determine the phenomenology of depressive symptoms in depressed adolescent inpatients reporting moderate to heavy alcohol consumption.

\section{Methods}

Subjects included 42 patients ( 30 girls, 12 boys) with DCM-III$\mathbf{R}$ major depression admitted to the University of Michigan Adolescent Psychiatry Inpatient Unit. Diagnostic information was

From the Department of Psychiatry, University of Michigan, Ann Arbor, MI. Address reprint requests to Cheryl A. King, Ph.D.; Department of Psychiatry; 200 East Hospital Drive; Ann Arbor, MI 48109-0290.

Dr. Shain is currently in private practice in Toledo, Ohio.

Received July 23, 1992; revised November 20, 1992. obtained from clinical interviews with adolescent and parent as well as 24- $h$ behaviorai observation. A subset of adolescents was interviewed using the Schedule for Affective Disorders and Schizophrenia for School-Age Children, Present Episode [KSADS-P; Puig-Antich and Ryan 1986; $n=18$ (43\%)]. Evaluation information was reviewed in a multidisciplinary case conference and diagnoses were established by consensus of the program director and the child psychiatry fellow. Patients ranged in age between 12.9 and 18.4 years (mean $=15.7$ years). Their socine:connmic status (SES) (Hollingshead and Redlich 1958) was distributed across levels (I: $9.5 \%$; II: $11.9 \%$; III: $21.4 \%$; IV: 35.7\%; V: 21.4\%).

Depression severity was rieasured with total scores on the Hamilton Ratirig Scale for Depression (HRSD; Hamilton 1960) and the Children's Depression Rating Scale-Revised (CDRSR; Poznanski et al 1984). The HRSD and the CDRS-R have been used extensively in child and adolescent research (King et al 1993; Shain et al 1991). These instruments are highly intercorrelated and have been validated using standardized diagnostic interviews and self-report measures of child and adolescent depression (Shain et al 1990). The CDRS-R was scored in terms of four subscales, the Mood, Somatic, Subjective, and Behavior scales, providing a symptom profile for each adolescent.

Quantity and frequency of alcohol consumption were assessed with the alcohol use section of the Diagnostic Interview for Children and Adolescents (DICA; Herjanic and Campbell 1977). The DiCA is a structured interview that was completed on all subjects by a trained nurse. Alcohol consumption was classified as None/Minimal (0-1 drinks/any frequency or 2-3 drinks/less than once monthly) or Moderate/Heavy (a fifth or more/any frequency or $>2$ drinks/at least once monthly). Twenty-five patients reported none/minimal alcohol use; 17 reported moderate/heavy use. There were no differences in the distribution of gender and SES between alcohol-consumption groups. The Moderate/Heavy consumption group, however, was significantly older than the None/Minimal group $[16.6 \pm 1.0$ versus $15.1 \pm 1.3$ years; $t$ (40) $=4.06, p \leqslant 0.001]$.

Comparisons between Moderate/Heavy and Nore/Minimal alcohol consumption groups were conducted using $t$-tests (total depression severity), univariate $F$ tests (specific symptom severity), $X^{2}$, Fisher's exact tests (presence of preceding dysi:- $-j$ - 
Table 1. Alcohol Consumption and CDRS-R Scores (Means and SD) among Depressed Adolescent Inpatients

\begin{tabular}{llc}
\hline & \multicolumn{2}{c}{ Alcohol consumption } \\
\cline { 2 - 3 } & $\begin{array}{c}\text { None/minimal } \\
(n=25)\end{array}$ & $\begin{array}{c}\text { Moderate/heavy } \\
(n=17)\end{array}$ \\
\hline CDRS-R subscales $^{a}$ & & \\
Mood & $15.3(4.7)$ & $15.6(4.8)$ \\
Somatic & $16.4(5.0)$ & $19.5(5.3)^{c}$ \\
Subjective & $12.6(4.5)$ & $14.3(5.5)$ \\
Behavior & $9.5(4.3)$ & $13.1(3.8)^{e}$ \\
CDRS-R items & & \\
School work & $3.4(2.1)$ & $5.2(2.1)^{c}$ \\
Capacity for fun & $3.3(1.7)$ & $4.4(1.2)^{d}$ \\
Social withdrawal & $2.8(1.7)$ & $3.5(1.4)$ \\
Sleep & $2.4(1.6)$ & $3.5(1.5)$ \\
Appetite & $2.8(1.7)$ & $3.2(1.7)$ \\
Fatigue & $4.1(2.0)$ & $5.4(1.6)^{d}$ \\
Physical complaints & $3.0(2.0)$ & $3.5(1.9)$ \\
Irritability & $3.9(1.4)$ & $4.1(1.7)$ \\
Guilt & $2.3(1.4)$ & $3.1(1.5)$ \\
Self-estecm & $4.0(1.3)$ & $3.9(1.7)$ \\
Depressed feelings & $3.6(1.6)$ & $4.7(1.9)^{d}$ \\
Morbid ideation & $2.4(1.4)$ & $3.3(1.6)^{c}$ \\
Suicidal ideation & $3.9(2.5)$ & $4.2(2.3)$ \\
Weeping & $4.2(2.3)$ & $3.7(2.2)$ \\
Depressed affect & $3.6(1.6)$ & $3.5(1.3)$ \\
Speech & $1.5(0.7)$ & $1.8(1.3)$ \\
Hypoactivity & $1.6(0.9)$ & $2.2(1.3)^{d}$ \\
\hline
\end{tabular}

${ }^{a}$ Overall multivariate $\boldsymbol{F}$ for the consumption effect on CDRS-R subscales significant, $F(4,37)=2.64, p<0.05$.

${ }^{b}$ Overall multivariate $F$ for the consumption effect on CDRS-R items nonsignificant.

${ }^{c} p<0.10:{ }^{a} p<0.05 ;{ }^{p} p<0.01$.

mia), and multivariate analyses of variance (overall profile of depressive symptoms).

\section{Results}

Subjects in the Moderate/Heavy alcohol consumption group were more depressed than those in the None/Minimal alcohol consumption group [CDRS-R: $62.4 \pm 14.2$ versus $53.8 \pm 13.2$, $t(40)=2.01, p=0.051$; HRSD: $21.3 \pm 7.4$ versus $17.0 \pm$ 6.0, $t(40)=2.10, p=0.042$ ]. As indicated in Table 1, patients in the Moderate/Heavy consumption group had higher scores than the None/Minimal consumption group on the CDRS-R Behavioral subscale, which includes anhedonia, social withdrawal, and schoolwork. Consistent with this fulding, the Moderate/Heavy consumption group had higher scores on five specific CDRS-R items: fatigue, depressive feelings, anhedonia, hypoactivity, and

\section{References}

Bukstein $\because \quad$ Brent DA, Kaminer Y (1989): Comorbidity of substance abuse and other psychiatric disorders in adolescence. Am J Psychiatry 146:113,1-1141.

Deykin EY, Levy JC, Wells V (1987): Adolescent depression, school difficulties. Forty-one percent of Moderate/Heavy users had preceding dysthymia compared to only $4 \%$ of other depressed inpatients $\left[\mathrm{X}^{2}(1)=6.82, p=0.009\right.$, with Yates correction].

Differences in depressive symptoms between alcohol consumption groups were not caused primarily by age differences. A median split was used to divide depressed inpatients into younger âid olủer ag̣e groups. Neither CDRS-R nor HRSD total scores differed by age group $[57.0 \pm 13.0$ versus $57.5 \pm 15.5, t(40)$ $=0.10$ and $18.2 \pm 6.7$ versus $19.3 \pm 7.1, t(40)=0.54$, respectively]. Differences between age groups on CDRS-R subscale and item scores were also nonsignificant, $F(4,37)=0.23$ and $F(17,24)=0.80$, respectively. Dysthymı was found to vary by age group with seven of eight inpatients diagnosed with dysthymia in the older age group (Fisher's Exact Test, $p=$ 0.04 ). Within the older age group, however, all dysthymic adolescents were in the Moderate/Heavy alcohol consumption group, supporting the hypothesized association.

\section{Discussion}

We found that the phenomenology of depression varied in depressed adolescent inpatients according to level of alcohol consumption. Moderate to heavy alcohol consumption was associated with prior dysthymia and greater severity of current symptoms, especially behavioral symptoms indicative of poor adaptive functioning. Several investigators have begun to study the comorbidity between depression and alcohol abuse in adolescents; however, this is the first study to our knowledge that addresses the phenomenology of depression in depressed adolescents who report heavy alcohol consumption. Our design does not permit us to infer causality as to whether alcohol misuse preceded, coincided with, or followed the development of depressive symptoms.

Because of the increased prevalence of alcohol abuse among depressed adolescents, a careful assessment of depression necessarily includes an assessment of possible coexisting substance abuse. Present findings suggest a depressive symptom profile that may serve as a "red flag" for alcohol abuse. That is, the presence of preceding dysthymia as weil as severe school difficulties, depressive feelings, fatigue, anhedonia, and hypoactivity, suggest the importance of an especially thorough and aggressive alcohol abuse assessment involving multiple informants. Early recognition and appropriate treatment of both comorbid conditions in adolescents with depression and alcohol abuse are essential.

This research was supported in pari by a Departmenta! Grant from the University of Michigan Department of Psychiatry. The authors thank the social workers and psychiatric nurses of the Adolescent Psychiatry Inpatient Program for assistance in data collection. We also thank Tamlynn Evans, Laurie McGovern, and Ana lacob for assistance in data management and Jackie Smith for assistance with manuscript preparation. alcohol and drug abuse. Am J Public Health 77:178182.

Greenbaum PE, Prange ME, Friedman RM, Silver SE (1991): Substance abuse prevalence and comorbidity with other psy- 
chiatric disorders among adolescents with severe emotional disturbances. J Am Acad Child Adolesc Psychiatry 30(4):575583.

Hamilton M (1960): A rating scale for depression. J Neurol Neurosurg Psychiatry 23:56-62.

Helzer JE, Pryzbeck TR (1988): The co-occurrence of alcoholism with other psychiatric disorders in the general population and its impact on treatment. $J$ Stud Alcohol 49:219-224.

Herjanic B, Campbell W (1977): Differentiating psychiatrically disturbed children on the basis of a structured interview. $J$ Abnorm Child Psychol 5:127-134.

Hollingshead AB, Redlich F (1958): Social class and mental illness. New York: Wiley.

King CA, Hill EM, Naylor MW, Evans T, Shain B (1993): Alcohol consumption in relation to other predictors of suicidality among adolescent inpatient girls. J Am Acad Child Adolesc Psychiatry 32(1):82-88.

Pfeffer CR, Newcorn J, Kaplan G, Mizruchi Mis, Mlutchik R
(1988): Suicidal behavior in adolescent psychiatric inpatients. J Am Acad Child Adolesc Psychiatry 27(3):357-361.

Poznanski EO, Grossman JA, Buchsbaum Y, Banegas M, Freeman L, Gibbons R (1984): Preliminary studies of the reliability and validity of the Children's Depression Rating Scale. J Am Acad Child Adolsc Psychiatry 23, 2:191-197.

Puig-Antich J, Ryan N (1986): Schedule for Affective Disorders and Schizophrenia for School-Age Children (6-18 years): Kiddie-SADS-Present Episode. Pittsburgh, PA: Western Psychiatric Institute and Clinic.

Regier DH, Farmer ME, Rae DS, et al (1990): Comorbidity of mental disorders with alcohol and other drug use. SAMA 264:2511-2518.

Shain BN, Naylor M, Alessi N (1990): Comparison of self-rated and clinician-rated measures of depression in adolescents. $\mathrm{Am}$ J Psychiatry 147:793-795.

Shain BN, King CA, Naylor M, Alessi N (1991): Chronic depression and hospital course in adolescents. I Am Acad Child Adolesc Psychiatry 30:428-433. 